\title{
Skin Adnexal Tumors: A study of 26 cases
}

\author{
Vidya Viswanathan*, Arpana Dharwadkar, Shruti Vimal, Parul Bhandari, Aditi Malhotra and Barnali Paul \\ Dr. D.Y. Patil Medical College Hospital and Research Center, Dr. D. Y. Patil Vidyapeeth, Pimpri, Pune
}

\begin{abstract}
Background: Skin adnexal tumours are a category of rare tumours. They usually present as painless nodules and papules. The diagnosis of these tumours poses a challenge, because there is usually a discrepancy in clinical and histopathological diagnosis.

Aims: To study the skin biopsies received in our department and compare their clinical and histopathological diagnosis.

Material and Methods: Prospective study of skin biopsies received in our department over a period of 1 year. The biopsies were processed and stained with haematoxylin and eosin and studied under the light microscope.

Results: Out of the 26 cases studied, 25 were found to be benign tumours with a single case of keratoacanthoma. The most common tumour encountered was pilomatricoma and the least common was syringoma. Only four cases showed clinical correlation with histopathological diagnosis.

Conclusion: It can be therefore concluded that skin adnexal tumours are rare tumours which need histopathological examination for their accurate diagnosis.
\end{abstract}

\section{Keywords: Adnexal Tumours, Rare, Histopathological Diagnosis}

\section{Introduction}

Adnexal tumours includes a wide spectrum of skin epithelial tumours which include hamartomatous lesions, hyperplasia, benign and malignant tumours that originate from or show differentiation toward adnexal structures, such as pilosebaceous unit, eccrine, and apocrine glands ${ }^{[1]}$. These tumours arise from multipotent stem cells which are present within epidermis or its appendageal structures. During neoplastic transformation, these tumors may aberrantly express one or more lines of appendageal differentiation of varying degree ${ }^{[1]}$. Adnexal skin tumors are usually misdiagnosed clinically, and histopathology provides us with diagnostic confirmation. Adnexal Tumors may display more than one line of differentiation, hence making precise classification of these tumors difficult. The diagnosis of these mixed tumors relies on histological evaluation, and they are usually classified according to the predominant morphological component seen histologically ${ }^{[2,3]}$. Adnexal Tumors are a group of skin tumors with are mostly benign in nature ${ }^{[2,3]}$. These tumors are usually found as solitary, sporadic lesions however, certain specific types of multiple tumors maybe an indication of some complex genetic syndromes for example, trichilemmomas in Cowden's syndrome and sebaceous tumours in Muir Torre syndrome ${ }^{[2,3]}$.

A malignant counterpart of almost every adnexal tumor has been seen. These tumours are rare, locally aggressive, and have the potential for nodal involvement and distant metastasis and hence have a poor clinical outcome. Therefore, establishing a diagnosis of malignancy in adnexal tumors is important for therapeutic, diagnostic and prognostic purposes ${ }^{[3]}$. In this article, we reviewed the histological features of the common skin adnexal tumors that we received in our institution, with an emphasis on diagnostic histological approach. We analysed adnexal tumours of the skin for their morphological, clinical, and histological features.

\section{Materials and methods}

The present study is a prospective study over a period of one year from Jan 2019 to Jan 2020. 26 cases which were diagnosed histopathologically as adnexal tumours were studied in Department of pathology at Dr. D. Y. Patil Medical College and Hospital, Pune, Maharashtra. Patient's clinical details such as age, sex, provisional clinical diagnosis, size, and location of the tumor were documented. The excised specimens were subjected to gross and microscopic examination. The histopathological examination was done on formalin fixed tissues and paraffin embedded blocks was made. Haematoxylin and Eosin stained sections were examined and few special stains like PAS and reticulin were performed wherever required. The tumors were classified according to predominant pattern of differentiation into follicular, sebaceous, eccrine, and apocrine tumors. 


\section{Results}

In our present study benign adnexal tumors constituted 25 out of 26 cases with one case of keratoacanthoma, this was in concordance with other studies where they found benign tumors to be more common as compared to malignant tumors. The cases were divided into tumors of sweat gland differentiation, hair follicle differentiation and sebaceous gland differentiation. In our study the tumors of the sweat gland differentiation were more commonly seen constituting 15 out of 26 cases. The rest of the tumors were of hair follicle differentiation constituting 11 out of 26 cases, this was in contrast to study conducted by other authors where they found tumors of follicular differentiation to be more common. We did not diagnosis any case of sebaceous gland differentiation in our study (table 1). The male to female ratio was found to be 1.8:1. These tumors had a significant male preponderance. The tumors were seen in all age groups ranging from $10-70 y r s$ with a peak seen in the third decade of life that is $21-30 y r s$ ( $9 / 26$ cases )( table 3 ). The extremities were the most common location for the tumor (15/26) followed by head and neck (7/26) and trunk (4/26) (table 2). Most of the tumors were small in size measuring $<1 \mathrm{~cm}$ in size $(16 / 26)$ with only two tumors presented with swelling measuring $>5 \mathrm{cms}$. Most of these tumors were not diagnosed clinically as adnexal tumors but as keratinous cysts as they presented as painless nodules and papules.

The sweat gland tumors in our study comprised of hideradenoma papilliferum, syringocystadenoma papilliferum, cylindroma, chondroid syringoma, eccrine poroma and syringoma. The most common sweat gland tumor encountered is hideradenoma papilliferum. The hair follicle tumors composed of pilomatricoma, trichoepithelioma and keratoacanthoma. The most common being pilomatricoma. There were no cases of tumors of sebaceous differentiation in the present study (table 4).

Table 1: Topographic distribution of lesions.

\begin{tabular}{|c|c|c|c|}
\hline Sr No: & Type of differentiation & No: of cases & Percentage incidence \\
\hline 1 & Sweat Gland & 15 & $57.67 \%$ \\
\hline 2 & Hair Follicle & 11 & $42.33 \%$ \\
\hline 3 & Sebaceous Differentiation & 0 & $0 \%$ \\
\hline Total & & $\mathbf{2 6}$ & $\mathbf{1 0 0 \%}$ \\
\hline
\end{tabular}

Table 2: Distribution of Cases based on site and gender.

\begin{tabular}{|c|c|c|c|c|c|}
\hline Sr No: & Site & Male & Female & Total & \% incidence \\
\hline 1 & Extremities & 10 & 5 & 15 & $57.69 \%$ \\
\hline 2 & Head \& Neck & 4 & 3 & 4 & $26.92 \%$ \\
\hline 3 & Trunk & 3 & 1 & $\mathbf{2 6}$ & $15.38 \%$ \\
\hline Total & & $\mathbf{1 7}$ & $\mathbf{9}$ & $\mathbf{1 0 0 \%}$ \\
\hline
\end{tabular}

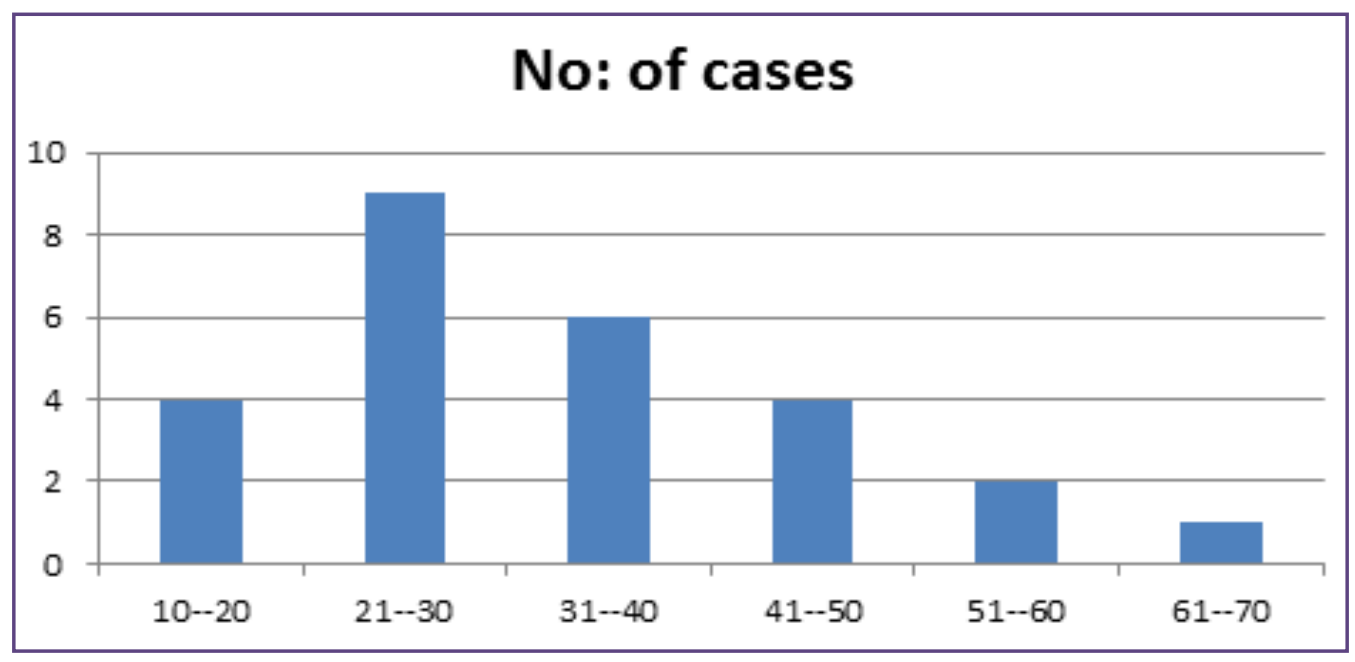

Fig. 1: Distribution of cases according to age. 


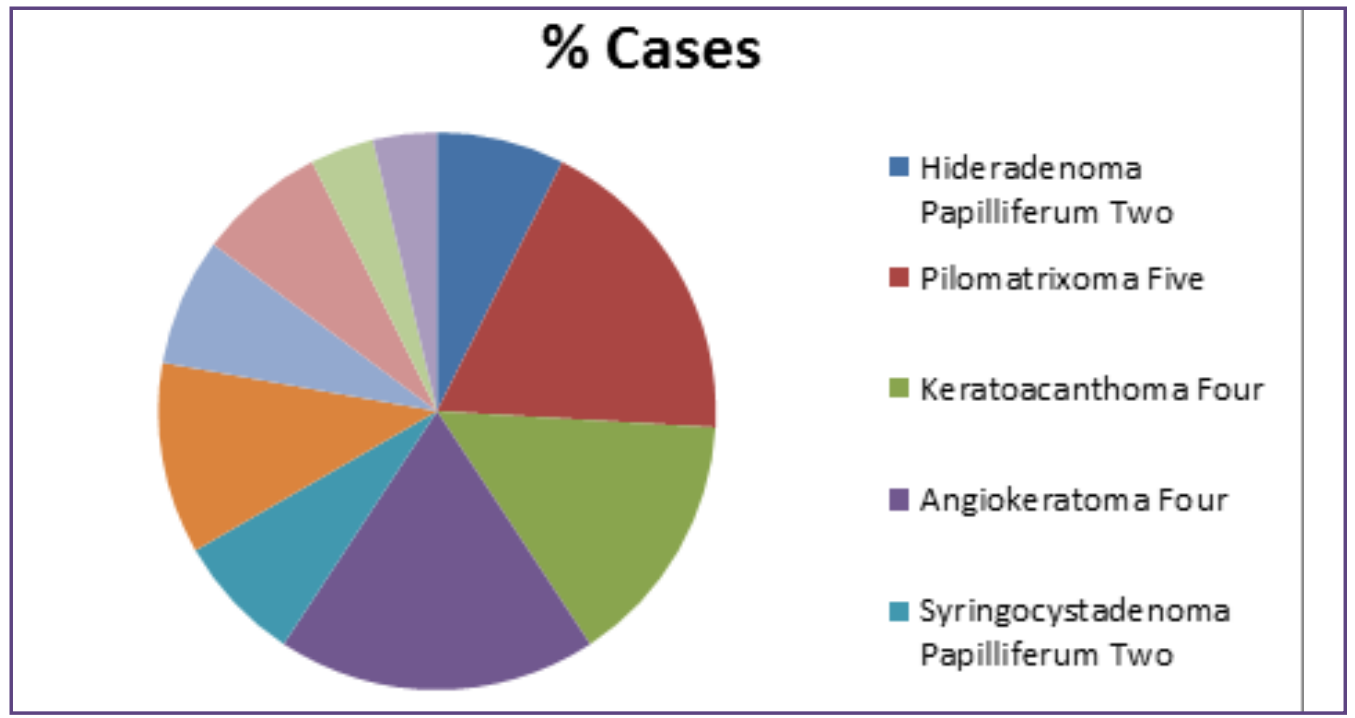

Fig. 2: Pie chart denoting distribution of cases.

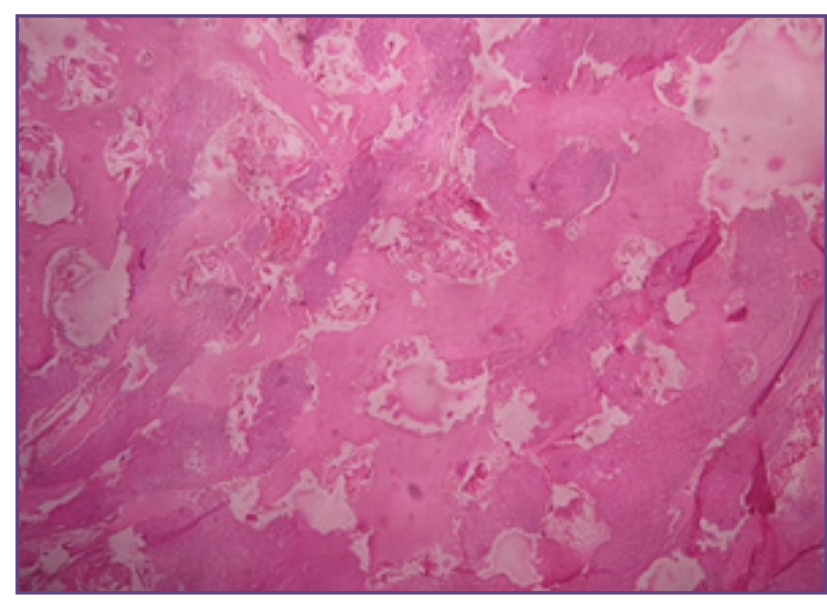

Fig. 3: Scanner view (4X) H\&E of Pilomatricoma.

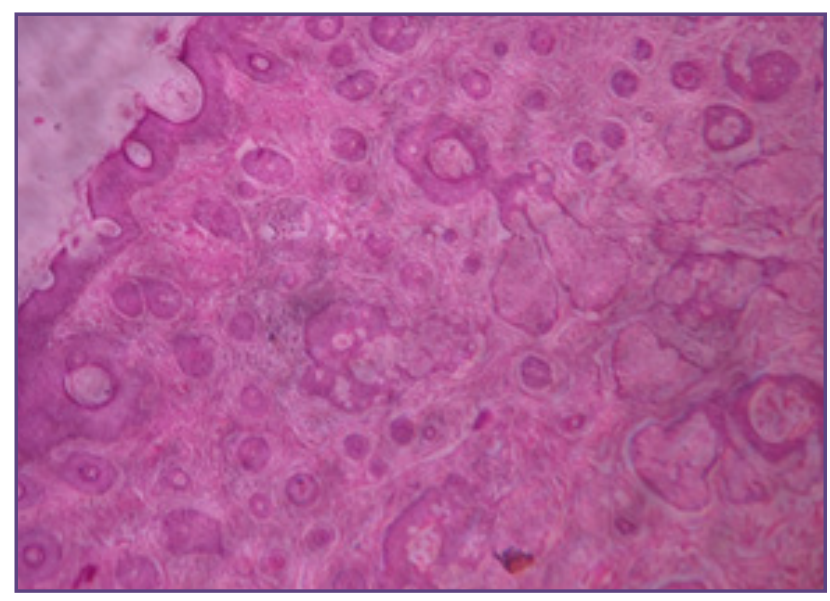

Fig. 5: Scanner view (4X) H\&E of Keratoacanthoma.

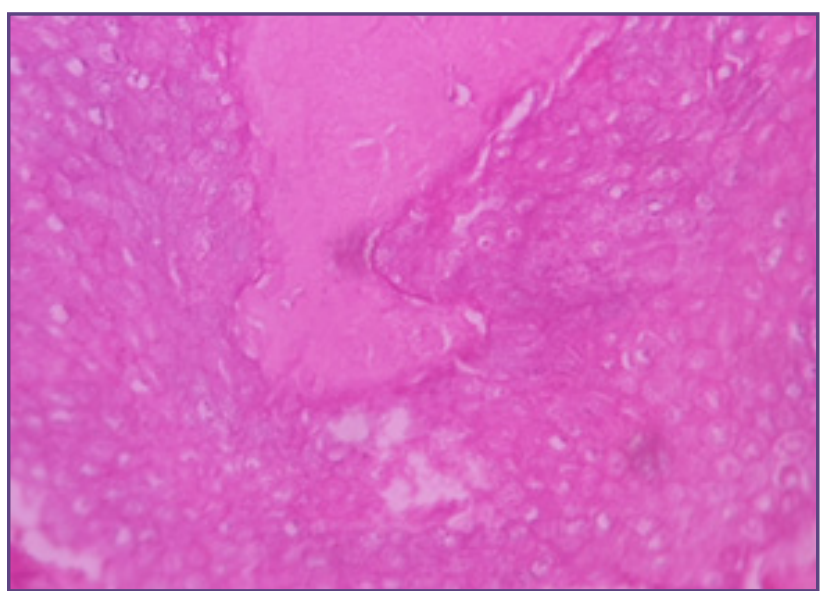

Fig. 4: High power view (40X) H\&E of Pilomatricoma showing ghost cells.

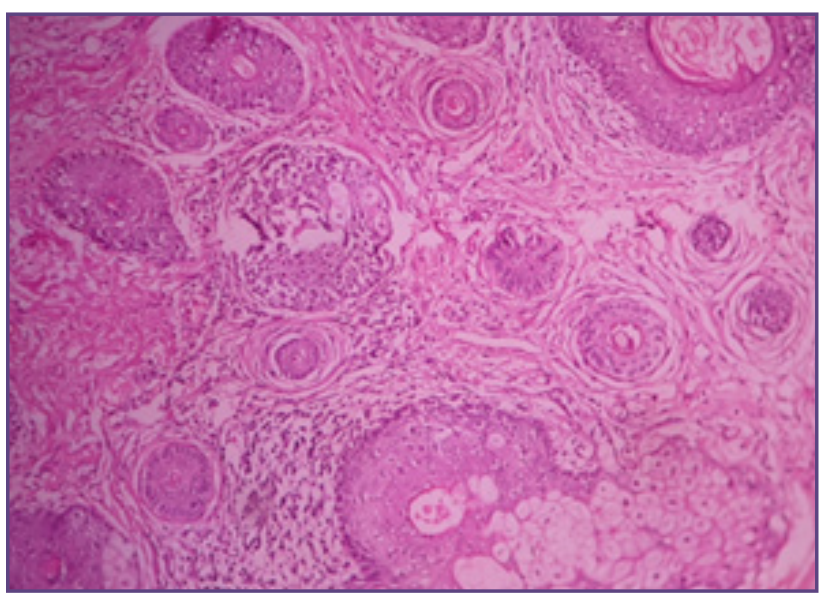

Fig. 6: High power view of Keratoacanthoma showing dome shaped nodules which have keratin filled central crater. 


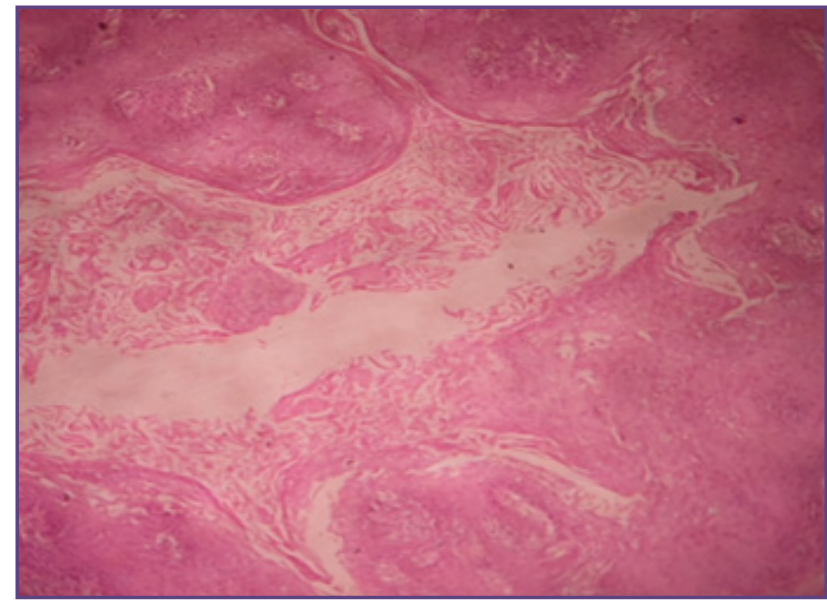

Fig. 7: Scanner view (4X) H\&E of Syringocystadenoma Papilliferum.

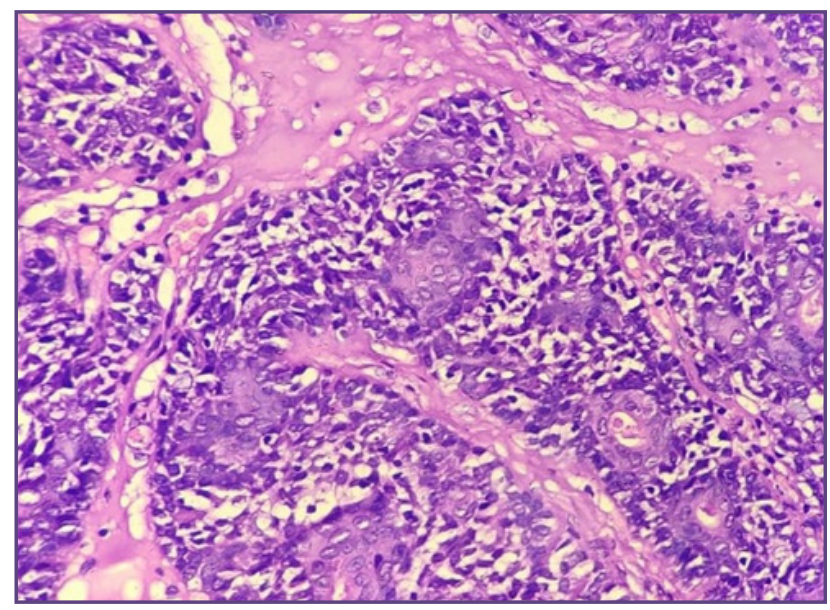

Fig.9: High power view (40X) H\&E of Hideradenoma paiplliferum.

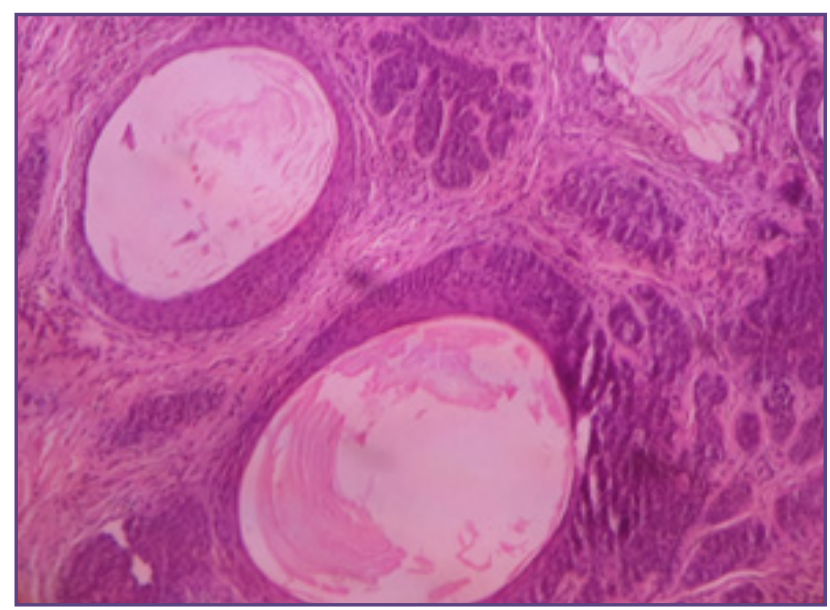

Fig.11: High power view(40X) H\&E of cylindroma.

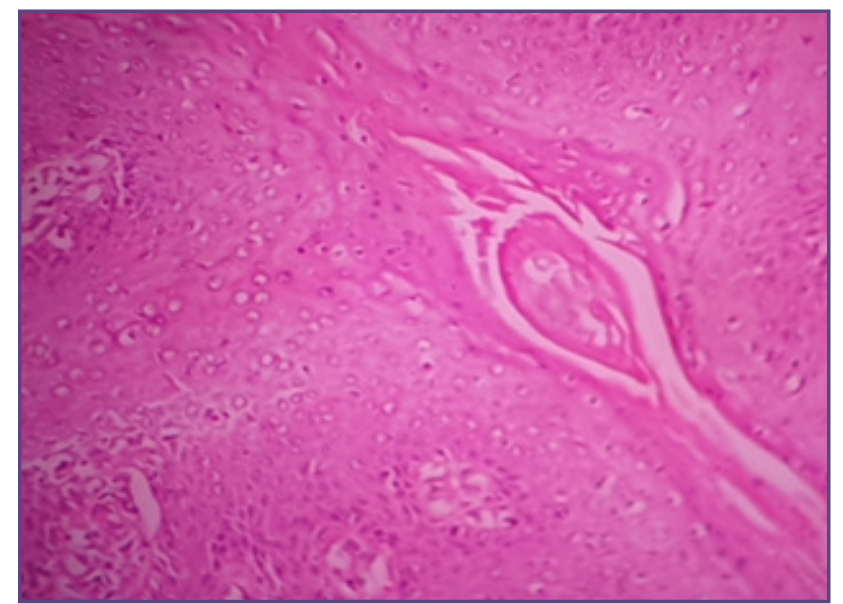

Fig. 8: High power view (40X) H\&E of Syringocystadenoma Papilliferum.

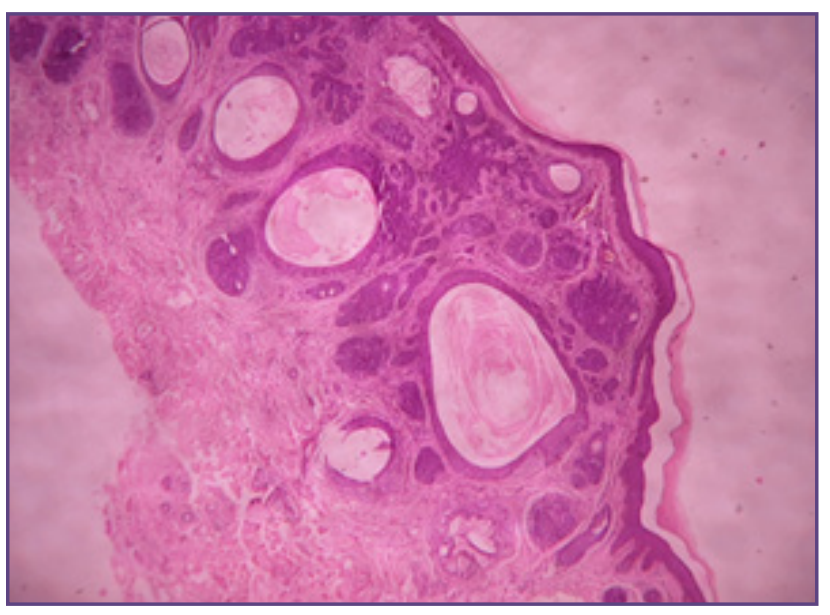

Fig.10: Scanner view(4X) H\&E of Cylindroma.

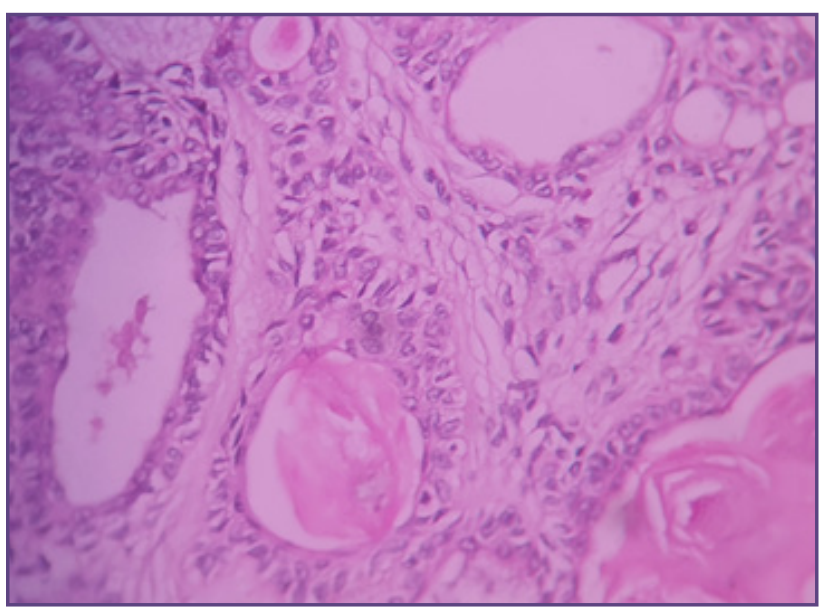

Fig. 12: High power view(40X) H\&E of Chondroid Syringoma. 


\section{Discussion}

Adnexal tumors are uncommon tumors which comprises of $<0.3 \%$ of all skin biopsies received ${ }^{[2,5,8]}$. Most of the tumors encountered in our study were benign lesions, which was in concordance with other studies such as Afthab et al. The most common tumor in our study was pilomatrixoma which is a benign hair follicle tumor. Similar findings were seen in study conducted by Kanwalpreet Kaur et al. These tumors accounted for $19.23 \%$ of all the tumors studied under adnexal tumors. Skin adnexal tumors are rare and histologically difficult to diagnose for a variety of reasons namely occurance of multiple lines of differentiation within the same tumor. These tumors arise from multipotential undifferentiated stem cells which have multiple differentiating pathways ${ }^{[2,7,8]}$. Adnexal tumors can be divided into three categories depending upon their line of differentiation. They are tumors of sweat gland differentiation, hair follicle differentiation and sebaceous gland differentiation. Adnexal tumors were thought to have a genetic basis of inheritance. The cell of origin was thought to be from either primary epithelial germ cells or cells of pre-existing structure. Mendelian inheritance and p53 mutations are important contributing factors in the genesis of adnexal tumors ${ }^{[11]}$.

Skin adnexal tumors need to be differentiated from squamous cell carcinoma and basal cell carcinoma. The traditional criteria of nuclear and cytological atypia alone are not enough to call a tumor malignant. Bernard Ackerman first emphasised the importance of architectural features in distinguishing between benign and malignant tumors. Benign tumors are vertically oriented and symmetrical with uniform epithelial cells, dense fibrotic stroma and absence of atypia, necrosis and mitosis. Whereas malignant tumors show asymmetry along with all the features mentioned above ${ }^{[1]}$.

The diagnosis of adnexal tumors is usually not suspected clinically. The usual presentation being flesh coloured nodules, papules and disfiguring lesions such as ulcers which are mistaken for keratinous cysts [2,7,9,11,15]. Histopathology remains the gold standard for the diagnosis of adnexal tumours as the clinical presentation is vague. Special stains such as period acid Schiff, mucicarmine, alcian blue and reticulin may help in arriving at a definitive diagnosis ${ }^{[2,7]}$.

The location of adnexal tumors is usually seen in the axilla, trunk and extremities. In our study we found the most common location to be the extremities. In other studies the head and neck region was found to be the most common location as they are rich in pilosebaceous units as seen by Punjani et al, Abanti et al, Sahu et al and Pachori et al. ${ }^{[2,7,8,14]}$.

Pilomatricoma was the most common tumor encountered in our study. Similar findings were noted by Kanwalpreet kaur et al and Alsaad et al. whereas Radhika et al found this tumor to be one of the rarely encountered adnexal tumors. It is a tumor of hair follicle differentiation. These are benign dermal or subcutaneous tumors most commonly seen in children and adolescents. It has a slight female preponderance. They have a predilection for head and neck region followed by upper extremities. Multiple pilomatricoma are usually associated with myotonic dystrophy, Rubinstein Taybi syndrome and Turner's syndrome. Classical pilomatricoma present as a well circumscribed nodular lesion in the dermis or subcutaneous tissue accompanied by fibrous stroma. The lesions are usually cystic and contain basaloid cells lining the cystic cavity which are continuously transformed into pale anucleated shadow or ghost cells admixed with keratin ( figure $3 \& 4$ ). The shadow cells are representative of failed attempts at hair shaft formation. Less commonly bone formation with extramedullary haematopoiesis maybe seen. Dystrophic calcification is also a common feature ${ }^{[3,9,11]}$.

Trichoepitheliomas are benign tumors which occur as solitary lesions on hair bearing skin with a predilection for head and neck. In our study the two cases of trichoepithelioma were seen around the nose which was in concordance with Nair et al. They also found trichoepithelioma to be the commonest adnexal tumor encountered which was in contrast to our study. It is an autosomal dominant disorder, the gene for which is associated with the short arm of chromosome 9. Histologically these are well circumscribed tumors, symmetric in nature with or without connection to the epidermis. They are composed of uniform basaloid cells showing peripheral palisading arranged in nests, trabaculae or cribriform patterns surrounded by dense stroma. The major differential diagnosis is basal cell carcinoma. Unlike $\mathrm{BCC}$ artifactual retraction is not seen in trichoepithelioma. Rarely a BCC may develop within the trichoepithelioma $[3,9,11]$.

Keratoacanthoma are symmetric, firm dome shaped nodules which have keratin filled central crater ( figure 5 \& 6 ). Histological examination showed buttressing of the epidermis over the side of the crater ${ }^{[12]}$. The study conducted by Afthab et al also showed that keratoacanthoma were rare tumors.

Syringocystadenoma Papilliferum are benign tumors of sweat gland differentiation. In our study we diagnosed four cases of this tumor, which was similar to the study 
conducted by Abanti et al who also diagnosed 4 cases. Among the four cases two presented in the second decade of life and the other two presented in the third decade of life. The cases showed a cystic lesion with papillary projections ( figure $7 \& 8$ ). Recent studies showed a loss of heterozygosity at chromosome 9q22 ${ }^{[7,9,16]}$.

Hideradenoma Papilliferum are characterised by medium sized round cells with hydropic change arranged in the form of nodules on light microscopy. Histopathologically most of these tumors are solid whereas cystic tumors have also been described ( figure 9 ). In our study of 26 cases four cases were diagnosed as hideradenoma papilliferum which was similar to study conducted by Radhika et al ${ }^{[11]}$.

Cylindroma are characterised by islands of tumor cells lined peripherally by small cells with dark nucleus. The tumor is surrounded by hyaline sheath ( figure 10 \& 11 ). Rarely do they become malignant. In our study two cases were diagnosed as cylindroma as seen by Nirali et al who also documented cylindroma to be one of the least common adnexal tumors ${ }^{[11,16]}$.

Chondroid syringoma presented as well circumscribed lobulated masses around the dermis or subcutaneous fat. They show prominent chondroid or myxoid stroma. The ducts are lined by epithelial myoepithelial bilayer and sometimes by keratinous cysts as seen by Nirali et al ${ }^{[16]}$. (figure 12)

Eccrine poromas are eccrine sweat gland tumors which usually occur in adults in the extrimeties. They can occasionally also show apocrine differentiation. Clinically they present as superficial plaques or nodules. Poromas involve both the epidermis and dermis. They show solid sheets of poroid cells with pushing borders ${ }^{[4,5]}$.

Syringomas these are tumors of sweat gland differentiation. The ducts of syringomas are composed of 1-2 layers of cuboidal cells rarely showing clear cell change ${ }^{[11,12]}$. Some of the ducts possess comma like tails which give the cell a tadpole like appearance as seen by Neela Patel et al and Nirali et al ${ }^{[13,16]}$.

\section{Conclusion}

The following conclusion can be drawn from the present study. Adnexal tumors are relatively rare with benign tumors outnumbering malignant tumors. In our study also most of the lesions studied were benign with only one case of malignant adnexal tumor. Tumors of eccrine differentiation formed the largest group followed by tumors affecting the hair follicle. There was a definite male preponderance with extremities being affected frequently. Most of these tumors presented in the third decade of life. Clinical correlation was not possible as clinical details were available for only a few cases. Even in those available they did not provide any information leading to a histopathological diagnosis of adnexal tumors. Hence histopathological diagnosis was the only tool used to diagnose these tumors. A large majority of these tumors are benign hence timely diagnosis and treatment such as excision can be curative in most cases.

\section{References}

1. Kanwalpreet Kaur, Karuna Gupta, Deepika Hemrajani, Ajay Yadav, and Kalpana Mangal. Histopathological Analysis of Skin Adnexal Tumours: A Three Year Study of 110 Cases at A Tertiary Care Center.Indian journal of dermatol.2017; JulAug;62(4):400-406.

2. M Pujani, GB Madaan,1 ZS Jairajpuri,2 S Jetley,2 MJ Hassan,2 and S Khan2. Adnexal Tumors of Skin: An Experience at a Tertiary Care Center at Delhi.Ann Med Health Sci Res. Sep-Oct 2016.

3. K O Alsaad, N A Obaidat, and D Ghazarian. Skin adnexal neoplasms-part 1: An approach to tumours of the pilosebaceous unit.J Clin Pathol.2007 Feb

4. Nidal A Obaidat, Khaled O Alsaad, and Danny Ghazarian. Skin adnexal neoplasms - part 2: An approach to tumours of cutaneous sweat glands. J Clin Pathol.2007 Feb;60(2): $145-159$

5. Alhumidi AA. Simple approach to histological diagnosis of common skin adnexal tumors. Pathology and laboratory medicine international;July 2017, volume 9;37-47

6. Ankit Sharma, Deepak G. Paricharak, Jitendra Singh Nigam , Shivani Rewri, Priyanka Bhatia Soni, Anita Omhare, and Preethi Sekar. Histopathological Study of Skin Adnexal Tumours-Institutional Study in South India. Journal of skin cancer 2014.

7. Abanti Saha, Nilay K Das, Ramesh C Gharami, Satyendra N Chowdhury, Pijush K Datta. A clinico-histopathological study of appendageal skin tumors, affecting head and neck region in patients attending the dermatology OPD of a tertiary care centre in Eastern India. Indian journal of dermatology 2011; vol 56;issue 1;page 33-36

8. Alaka Sahu, Dilip Kumar Sa, Salil Kumar Nayak and Kailash Chandra Agrawal. Skin Adnexal Tumors: A Histopathological Study of 60 Cases at a Tertiary Care Centre. Annals of pathology and laboratory medicine; vol 5 no $3 ; 2018$

9. Pradeep S Nair. Aclinicopathologic study of skin appendageal tumors. Indian journal of dermatology, venereology and leprology, 2008; vol 74; issue 5 ; page 550

10. Vijayan P, Nayak R. Spectrum of skin adnexal tumors with eccrine and apocrine differentiation - A single institution study of 40 cases with clinicopathological correlation. Journal of Pathology of Nepal (2015) Vol. 5, 727-732.

11. K. Radhika, B.V. Phaneendra, N. Rukmangadha, M.K. Reddy. A study of biopsy confirmed skin adnexal tumours: experience at a tertiary care teaching hospital. Journal of 
clinical and scientific research 2013; vol 2; issue 3 ; page $132-138$.

12. Afthab Jameela Wahab, V. Anandan, Mani Surya Kumar, P.S. Mohanasundari, Ashwini B. A variety of skin tumors arise from the epidermis, epidermal appendages, dermis and subcutis. Many a time clinical diagnosis becomes difficult, when histopathological examination (HPE) aids in arriving at the correct diagnosis. Indian journal of clinical and experimental dermatology 2017; vol 3; issue 3; page 96-100.

13. Neela Patel, Tarul Suthar, Hiren Suthar, Anisha Arora. A study of tumours, tumour like lesions and cysts of epidermis and its appendages. Indian journal of clinical and experimental dermatology 2018;vol 4; issue 3; page 194-200.
14. Geeta Pachori, Manisha Jain, Soniya Sanadhya, Tushar Bayla. A Study of Spectrum of Skin Adnexal Tumors in Ajmer Region. International journal of medical research professionals 2017; Sept; 3 (5); 84-87.

15. Dr. Jibran Amin, Dr. Lateef Ahmad Wani, Dr. Suhail Farooq, Dr. Baba Iqbal Khaliq and Dr. Farzana Manzoor.To study the histopathological patterns of skin adnexal tumors. International journal of current research 2017.

16. Nirali Amin, Smita Shah, Shreedhan Prajapati, Hansa Goswami. Histomorhological spectrum of skin adnexal tumors at a tertiary care hospital - A retrospective study. Int J Cur Res Rev 2016; vol8; issue 4.

*Corresponding author:

Dr Vidya Viswanathan, Dr. D.Y. Patil Medical College,

Phone: +91 9822046668

Email: docvidya11@gmail.com

Date of Submission : 28/06/2020

Date of Acceptance : 06/10/2020

Financial or other Competing Interests: None.

Date of Publication : 30/11/2020 